\title{
Arbeitsmarktpolitische Hürden zu einer Willkommenskultur
}

\author{
WILHELM ADAMY
}

Die Gründe für Migration und Zuwanderung sind vielfältig. Insbesondere die Zahl der Menschen, die vor politischer Verfolgung, Krieg oder Vertreibung nach Deutschland fliehen und hier politisches Asyl bzw. internationalen Schutz beantragen, ist in den letzten Jahren kontinuierlich gestiegen. Viele bleiben für längere Zeit oder sogar für immer als anerkannte Asylberechtigte oder Geduldete in Deutschland. Bisher stehen diese Menschen vor zahlreichen Barrieren, um ihren eigenen Lebensunterhalt sichern zu können. Die Wohnungsversorgung, das Erlernen der deutschen Sprache sowie die Eingliederung in Ausbildung und Beschäftigung sind von zentraler Bedeutung für eine soziale und gesellschaftliche Integration. Die Herstellung ökonomischer und gesellschaftlicher Teilhabechancen ist nicht nur eine humanitäre Verpflichtung, sondern auch längerfristig in unserem eigenen Interesse. Aus gewerkschaftlicher Sicht brauchen wir eine offene und transparente Einwanderungs- und Willkommenskultur. Zugleich muss aber auch das Augenmerk auf Geringqualifizierte und Langzeitarbeitslose gerichtet werden, die bisher nicht oder nur völlig unzureichend in Beschäftigung integriert werden konnten; auch sie gilt es noch besser zu fördern und ihre Arbeitsmarktchancen zu verbessern.

Die immer wieder geforderte Willkommenskultur muss auch von der Mehrheitsgesellschaft, von Unternehmen und staatlichen Institutionen mit Leben gefüllt werden. Dies gilt auch für die Arbeitsmarkt- und Bildungspolitik. Aus arbeitsmarktpolitischer Sicht befasst sich der drittelparitätisch besetzte Verwaltungsrat der Bundesagentur für Arbeit (BA) seit Längerem mit diesem Thema und will die Integration von Zuwanderern und Flüchtlingen in Ausbildung und Beschäftigung besser unterstützen.

So hat der BA-Verwaltungsrat 2014 den Austausch mit dem Bundesamt für Migration und Flüchtlinge (BAMF) gesucht, eine Fachtagung mit Bundes- und Länderministerien sowie anderen Netzwerkpartnern organisiert und eine Arbeitsgruppe eingesetzt, die ausbildungs- und ar- beitsmarktliche Handlungsempfehlungen für humanitäre Zuwanderung vorlegte. ${ }^{1}$ Eine möglichst frühzeitige Arbeitsmarktintegration für die steigende Zahl von Asylsuchenden und anderen Flüchtlingen ist wichtiges Element gesellschaftlicher Integration und kann der Gefahr lang anhaltender Arbeitslosigkeit entgegenwirken.

Doch einer besseren arbeitsmarktlichen Unterstützung und Integration von Flüchtlingen und Asylbewerbern stehen noch vielfältige Hindernisse im Weg.

\section{Bisheriger Arbeitsmarktzugang und Förderung}

$\mathrm{Ob}$ und inwieweit arbeitsuchende Zuwanderer und Asylberechtigte den Arbeitsagenturen oder den Jobcentern zugeordnet werden, hängt vom jeweiligen aufenthaltsrechtlichen Status und dem damit einhergehenden Arbeitsmarktzugang ab. $\mathrm{Zu}$ Beginn des Asylverfahrens ist in der jetzt auf drei Monate verkürzten Wartefrist bisher nur ein Zugang zur Beratung durch die Arbeitsagenturen möglich. Erst wenn der Arbeitsmarktzugang vorhanden ist, können Arbeitsagenturen vermitteln und arbeitsmarktbezogene Leistungen gewähren. Für Asylbewerber und Geduldete - soweit für Letztere kein generelles Arbeitsverbot gilt - ist dies nach drei Monaten Aufenthalt der Fall. Sie beziehen Leistungen nach dem Asylbewerberleistungsgesetz und können dann über die beitragsfinanzierte Arbeitslosenversicherung gefördert werden.

Anerkannte Flüchtlinge haben demgegenüber einen uneingeschränkten Arbeitsmarktzugang und beziehen - soweit sie hilfebedürftig sind Hartz-IV-Leistungen. Sie werden somit von den Jobcentern betreut sowohl hinsichtlich der passiven Leistungen wie der arbeitsmarktpolitischen Fördermöglichkeiten. Die vermittlungsrelevanten Handlungsmöglichkeiten und Integrationsmöglichkeiten unterscheiden sich damit auch für diese Zielgruppe danach, welchem System sie zugeordnet werden.
Einer nachhaltigen und qualifikationsadäquaten Arbeitsmarktintegration stehen oftmals rechtliche und organisatorische Hindernisse im Wege. Ein von der Arbeitslosenversicherung 2014 gestartetes Modellprojekt zur frühzeitigen Arbeitsmarktintegration von Asylbewerbern gibt vielfältige Hinweise für notwendige Weiterentwicklungen. So zeigen die dort gemachten Erfahrungen, dass diese Zuwanderergruppen spezifische Anforderungen an die Qualifikation der Vermittlungskräfte stellen und mit dem Arbeitsmarktzugang nach drei Monaten bisher oftmals noch keine qualifizierte Ausbildungs- oder Arbeitsmarktintegration möglich ist. Zugleich zeigen sich Förderlücken in beträchtlichem Aus$\mathrm{maß}$.

\section{Sprachkursangebot unzureichend}

Das Erlernen der deutschen Sprache ist eine zentrale Eintrittskarte für eine gelingende Integration. Das zur Verfügung stehende Sprachkursangebot ist aktuell aber qualitativ und quantitativ völlig unzureichend. Zwar gibt es bei der Sprachförderung abgestufte Fördermöglichkeiten mit unterschiedlichen Zuständigkeiten und Finanzierungsstrukturen, aber für Asylbewerber und Geduldete greift diese Förderkette nicht. Diese ausschließlich bundesfinanzierten Integrationskurse sollen allgemeinsprachliche Deutschkenntnisse vermitteln. Dieses Programm liegt in der Zuständigkeit des Bundesinnenministeriums und wird vom BAMF umgesetzt. Asylbewerber und Geduldete haben keinen Zugang zu diesen Integrationskursen, die vor allem allgemeinsprachliche Grundlagen vermitteln.

Als zweite Förderkette soll das ESF-BAMFProgramm berufsbezogene Deutsch- und Fachkenntnisse vermitteln, die oftmals Voraussetzungen einer arbeitsmarktpolitischen Eingliederung

1 Siehe Bundesagentur für Arbeit, Presse-Info vom 24.04.2015. 
sind. Dieses Programmelement liegt in der $\mathrm{Zu}$ ständigkeit des BMAS und wird ausschließlich aus EU-Mitteln finanziert. Dieses Angebot setzt Grundsprachkenntnisse voraus und reicht vom klassischen Unterricht unter Einbeziehung beruflichen Fachvokabulars bis zum konkreten Berufspraktikum im Betrieb. Der Förderzeitraum beträgt bei Vollzeitmaßnahmen höchstens sechs und bei Teilzeit zwölf Monate. Viele Asylbewerber verfügen jedoch nicht über die erforderlichen Grundkenntnisse, sodass ihnen oftmals die berufsbezogene Deutschförderung verschlossen bleibt. Finanzielle Mittelkürzungen erzeugen weitere Ausschlüsse.

Während in der ausgelaufenen ESF-Förderperiode noch eine Aufstockung auf rd. 310 Mio. $€$ erfolgte, stehen in der neuen ESF-Förderperiode - wegen der deutlich geringeren Mittelzuteilung seitens der EU-Kommission - für Deutschland nur noch 180 Mio. € zur Verfügung. Diese geringere Finanzausstattung hat spürbare Auswirkungen. So können - anders als im abgelaufenen Programm - Vorschaltkurse nicht mehr finanziert werden, mit deren Hilfe insbesondere Asylbewerbern und Geduldeten zunächst Grundsprachkenntnisse vermittelt werden. Sowohl bei den Integrationskursen wie dem ESF-BAMFProgramm gibt es eine beträchtliche rechtliche Förderlücke beim Erlernen der deutschen Sprache und von Fachkenntnissen, die durch Mittelkürzungen noch verschärft wird.

\section{Anerkennung ausländischer Berufsabschlüsse}

Auch bei der Anerkennung ausländischer Berufsabschlüsse zeigen sich Probleme. Zwar sieht das Bundesanerkennungsgesetz einen Rechtsanspruch auf eine Überprüfung der Gleichwertigkeit von im Ausland erworbenen Berufsabschlüssen vor. Doch das Gesetz enthält keine Regelungen zur Finanzierung der mit der Anerkennung verbundenen Kosten und Gebühren. Übersetzungen oder Beglaubigungen oder evtl. erforderliche Nachqualifizierungen müssen die Antragsstellenden grundsätzlich selbst übernehmen; nur wenn sie arbeitsuchend sind, können diese Kosten unter den üblichen gesetzlichen Voraussetzungen von Arbeitsagentur oder Jobcenter übernommen werden. Aber auch die Förderpraxis weist erhebliche regionale Unterschiede auf. So ist die Übernahme von Kosten im Anerkennungsverfahren von Fachkräften aus dem EUAusland eine Ermessensentscheidung der Vermittler, soweit die Betroffenen arbeitsuchend gemeldet sind.
Die größten Hürden im Anerkennungsverfahren bestehen bei der Kostenübernahme für die zuwandernden Fachkräfte aus Drittstaaten sowie für Asylbewerber in der Phase des Asylverfahrens, weil in diesen Fällen in der Regel keine arbeitsmarktpolitische Fördermöglichkeit besteht. Zudem gibt es eine erhebliche Spannbreite bei den entstehenden Kosten, und der Verwaltungsvollzug ist je nach Bedarf in den Ländern sehr unterschiedlich. Der BA-Verwaltungsrat setzt sich daher für Kostenkorridore und einen einheitlichen Verwaltungsvollzug in den Ländern ein.

\section{Arbeitsmarktpolitische Förderung}

Während des Asylverfahrens ist in der dreimonatigen Wartefrist aktuell nur ein Zugang zur Beratung durch die Arbeitsagenturen möglich. Erst wenn Arbeitsmarktzugang besteht, können Arbeitsagenturen oder Jobcenter durch Vermittlung und bestimmte arbeitsmarktliche Hilfen unterstützen. Um möglichst früh zu beraten und Informationen zu den vorhandenen Kompetenzen und Berufserfahrungen gewinnen zu können, unterstützt die Arbeitslosenversicherung das Modellprojekt zur frühzeitigen Arbeitsmarktintegration von Asylbewerbern („Early Intervention"). Die dort gemachten Erfahrungen zeigen, dass mit dem Arbeitsmarktzugang nach drei Monaten vielfach noch keine qualitativ gute Integration in Ausbildung und Arbeitsmarkt möglich ist. Zudem stellen sich hier relativ hohe Anforderungen an Qualifikation und Flexibilität der Vermittlungsfachkräfte. Der IAB-Praxisbericht ${ }^{2}$ zeigt Handlungsbedarf insbesondere in folgenden Punkten:

Die Betreuung von Asylbewerbern macht es erforderlich, dass Vermittlungsfachkräfte möglichst über zusätzliche Kenntnisse wie Fremdsprachen oder spezifische Rechtskenntnisse verfügen - wie bspw. zum Aufenthaltsrecht. Informationen und Kontakte zu Bleiberechtsnetzwerken sind gleichfalls sinnvoll. Eine Herausforderung kann ebenso die Klärung von beruflicher Qualifikation und Erfahrung sowie beruflichen Vorstellungen der Teilnehmenden sein. Gesprächsdiagnostik und erweiterte Möglichkeiten der Kompetenzfeststellung im Rahmen der Vermittlungsgespräche sind hilfreich.

Ein Rechtskreiswechsel vom Versicherungsins Fürsorgesystem kann gleichfalls zu Brüchen in der Integrationsstrategie führen. So sind die Fachkräfte in den Jobcentern nicht daran gebunden, die zuvor von der Arbeitsagentur eingeleitete Integrationsstrategie fortzusetzen. Nicht zu- letzt aufgrund der ohnehin völlig unzureichenden Fördermöglichkeiten sind Kontroversen über die Ausfinanzierung eingeleiteter Maßnahmen keinesfalls auszuschließen. Um den Wechsel möglichst friktionsfrei gestalten zu können, ist ein rechtskreisübergreifendes Übergabemanagement bzw. Handlungshilfen anzustreben.

Rechtliche Unsicherheiten bestehen ebenso hinsichtlich des Umfangs der zur Verfügung stehenden Förderinstrumente. So können Asylbewerber und Geduldete nur sehr eingeschränkt ausbildungsvorbereitende und begleitende Hilfen in Anspruch nehmen. Die rechtliche Beschränkung auf die Förderung einer betrieblichen Ausbildung sollte aufgegeben werden. Zugleich sollte sichergestellt werden, dass für junge Flüchtlinge und Geduldete der Aufenthalt während und unmittelbar nach Ausbildung gesichert ist und sie in dieser Zeit ihr Aufenthaltsrecht nicht verlieren.

Unzureichende Deutschkenntnisse und fehlende Teilnahmechancen an Integrationskursen und aufbauendem ESF-BAMF-Programm verhindern oder erschweren zugleich arbeitsmarktpolitische Fördermöglichkeiten - wie berufliche Weiterbildung. Arbeitsmarktpolitische Fördermöglichkeiten drohen leer zu laufen, denn nur mit ausreichenden allgemeinen und berufsbezogenen Deutschkenntnissen kann Weiterbildung wirksam sein und der Sprung in die Arbeitswelt gelingen. Hohe Folgekosten drohen, wenn aufgrund unzureichender Deutschkenntnisse die Integration in Arbeitsmarkt und Gesellschaft verzögert wird oder gar misslingt. Es ist daher dringend geboten, Flüchtlingen mit Bleiberechtsstatus sowie Personen mit einer hohen Bleibeperspektive eine Deutschförderung im Rahmen der Integrationskurse wie dem ESF-BAMF-Programm zu eröffnen.

\section{Finanzielle Auswirkungen}

Die steigenden Asylbewerberzahlen sowie ein schnellerer Arbeitsmarktzugang haben finanzielle Auswirkungen. Auch wenn sich dieser Personenkreis längst nicht immer arbeitslos melden wird, zeigt sich der arbeitsmarktpolitische Handlungsbedarf von Agenturen und Jobcentern sowohl beim Personal wie den arbeitsmarkt-

2 Vgl. Daumann, V./Dietz, M./Knapp, B./Strien, K. (2015): Early intervention - Modellprojekt zur frühzeitigen Arbeitsmarktintegration von Asylbewerberinnen und Asylbewerbern. Ergebnisse der qualitativen Begleitforschung: Institut für Arbeitsmarkt- und Berufsforschung, IAB-Forschungsbericht (3), Nürnberg. 
politischen Fördergeldern. In den Jobcentern und Arbeitsagenturen werden derzeit rund 360.000 Asylbewerber und Flüchtlinge betreut, von denen etwa die Hälfte beruflich qualifiziert ist.

Die Beitragszahler zur Arbeitslosenversicherung übernehmen bereits heute finanzielle Verantwortung für diese gesamtgesellschaftliche Aufgabe wie das Modellprojekt zur frühzeitigen Arbeitsmarktintegration von Asylbewerbern exemplarisch zeigt. Jetzt geht es im Versicherungssystem darum, den notwendigen Personalbedarf bereitzustellen und die haushaltsrechtlichen Voraussetzungen für eine bedarfsgerechtere Arbeitsmarktförderung zu schaffen sowie sicherzustellen, dass dies nicht zulasten der Fördermöglichkeiten anderer Personengruppen geht.

Im Hartz-IV-System müsste dies vom Bund gleichermaßen gewährleistet werden. Denn die qualitative Personalausstattung wie die generelle Mittelausstattung im Hartz-IV-System sind bisher völlig unzureichend. Es muss daher sichergestellt werden, dass die Fördermöglichkeiten für Langzeitarbeitslose und Geringqualifizierte wie für hilfebedürftige Flüchtlinge gleichermaßen verbessert werden. Anderenfalls droht sich die ohnehin schwierige Arbeitsmarktsituation dieser beiden Personengruppen nochmals zu verschlechtern. Der Bundesfinanzminister ist hier in besonderer Weise gefordert. Dies gilt auch hinsichtlich der Sprachförderung. Allein für eine bedarfsgerechte Ausgestaltung der Integrationskurse sind mindestens 300 bis 400 Mio. $€$ jährlich erforderlich. Neben der Überwindung von Förderlücken und der Einbeziehung von Flüchtlingen im Asylverfahren sollten auch EU-Bürger einbezogen werden können; zudem müsste die Vergütung der Lehrkräfte in diesen Kursen erhöht werden, die bisher meist prekär und auf Honorarbasis beschäftigt werden.

Auch im ESF-BAMF-Programm sind zusätzliche Steuermittel erforderlich, da bei geringeren Finanzmitteln Asylbewerber und Geduldete nur sehr begrenzte Teilnahmemöglichkeiten haben. Auch wenn die Fakten seit Langem auf dem Tisch liegen und sich Investitionen in die Sprachförderung von Zuwanderern längerfristig auszahlen, ist der Bund hier finanziell immer noch sehr restriktiv, ebenso hinsichtlich einer besseren finanziellen Ausstattung der Arbeitsförderung im Hartz-IV-System.

\section{Fazit}

In jüngster Zeit gibt es einige gesetzliche Erleichterungen beim Arbeitsmarktzugang für die gestiegene Zahl von Asylbewerbern und Flüchtlingen. Die Bemühungen zur besseren Arbeitsmarktintegration haben damit nicht Schritt halten können. Nach wie vor gibt es hohe Hürden, die einer gelebten Willkommenskultur entgegenstehen. Eine zentrale Voraussetzung für eine bessere gesellschaftliche und arbeitsmarktpolitische Integration von Asylbewerbern und Flüchtlingen sind bedarfsgerechte Angebote zum Erlernen der deutschen Sprache. Doch genau hier hapert es, weil nicht genügend Steuermittel zur Verfügung gestellt werden.

Aktuell gibt es immer noch beträchtliche Förderlücken bei der allgemeinen und berufsbezogenen Sprachförderung. Diese Hürden müssten dringend abgebaut und Steuermittel von mindestens 300 bis 400 Mio. € zusätzlich pro Jahr zur Verfügung gestellt werden. Werden diese Mittel nicht bereitgestellt, droht ein Vielfaches an Folgekosten. Notwendig ist nicht nur eine Mentalitätsveränderung, sondern ein politischer Perspektivwechsel. Flüchtlingen mit Bleiberechtsstatus sowie Menschen, die absehbar auf längere Zeit in Deutschland bleiben werden, müssen bessere Integrationschancen eröffnet werden. Hierfür ist eine gesamtgesellschaftliche Haltung, die Flüchtlinge willkommen zu heiBen, zwar notwendig, aber allein nicht ausreichend vielmehr müssen auch Strukturen geschaffen und die notwendigen finanziellen Mittel bereitgestellt werden, die ihre Integration fördern. Insbesondere der Bund ist hier gefordert. Zusätzliche Steuermittel sind bei der Sprachförderung notwendig; zudem ist das steuerfinanzierte Hartz-IV-System genauso gefordert wie die beitragsfinanzierte Arbeitslosenversicherung. Beide Institutionen müssen die soziale und arbeitsmarktpolitische Integration von Flüchtlingen stärker in den Blick nehmen. Dabei sollen insbesondere für Frauen - auf ihre spezifische Problemlage ausgerichtete - Unterstützungsangebote einbezogen werden.

Beim Flüchtlingsgipfel im Kanzleramt am 8. Mai 2015 ist bisher noch nicht viel herausgekommen. Lediglich das BAMF bekommt mehr Personal zur Beschleunigung der Verfahren. Zur Sprachförderung sollen offensichtlich in diesem Jahr nur 25 Mio. $€$ zur Verfügung gestellt werden, während im Hartz-IV-System bislang keine zusätzlichen Mittel vorgesehen sind. Die Bereitschaft der Bundesregierung zur Umsetzung und Finanzierung der Leitlinien des BA-Verwaltungsrates zur besseren Arbeitsmarktintegration von Asylbewerbern scheint gering.

\section{AUTOR}

WILHELM ADAMY, Dr., ist Abteilungsleiter für Arbeitsmarktpolitik beim Deutschen Gewerkschaftsbund (DGB) und Sprecher der Gewerkschaftsgrup pe im BA-Verwaltungsrat. Arbeitsschwerpunkte: Arbeitsförderung und Berufliche Weiterbildung, Ordnung auf dem Arbeitsmarkt sowie Finanzierung des Sozialstaates.

wilhelm.adamy@dgb.de 